\author{
Editorial Communication
}

\author{
RETRACTED
}

\title{
THE EFFECT OF HMW-GS ON GLU-A1, GLU-B1, GLU-D1 IN SOME LOCAL BREAD (Triticum aestivum L.) WHEAT GENOTYPES
}

This article has been retracted after publication and DOI registration by the Editorial

Board because of antagonistic differences between statements of authors after publication. 